International Journal of Wireless Communications and Networking Technologies

Available Online at http://warse.org/IJWCNT/static/pdf/file/ijwcnt01942020.pdf

https://doi.org/10.30534/ijwent/2020/01942020

\title{
Fingerprint Identification in Infants
}

\author{
Bhavana R Maale ${ }^{1}$, Sameena Kauser ${ }^{2}$ \\ ${ }^{1}$ Assistent Professor Dept. Of Computer Science Engineering, VTU Regional Office, Kalaburagi, India \\ sg.bhavana@gmail.com \\ ${ }^{2}$ Student, Dept. of Computer Science and Engineering, VTU Regional Office, Kalaburagi, India \\ sameenakauser32@gmail.com
}

\begin{abstract}
Children square measure progressively stringent biometrics- recognition for variety of applications, significantly in developing countries wherever kids don't have any variety of identification. Such tasks embody observation child vaccination schedules, detecting missing babies, preventing food grant fraud and preventing in- babe swaps. Our objective is to develop a fingerprint-based identification system for infant (age range: 0-12 months). Our current analysis has addressed the subsequent issues: (i) style of a compact, easy, high-resolution (>1,000 ppi) fingerprint reader; (ii) image improvement algorithms to boost quality of child fingerprint images; and (iii) assortment of longitudinal infant fingerprint information to calculate identification accuracy over time. Here we have a tendency to planned a crucial step in automatic fingerprint matching is to mechanically and faithfully extracted trivia from input fingerprint picture. However, the performance of a trivia extraction rule depends heavily on the standard of the input fingerprint pictures. So as to make sure that the performance of associate automatic fingerprint identification/verification system would be strong with reference to the standard of the fingerprint picture, it ought to be essential to include a fingerprint improvement rule within the trivia extraction module. In our case, the standard of the image is absolutely smart, and that we ought not to enhance our image.
\end{abstract}

Key words: Fingerprint, Biometric, image enhancement

\section{INTRODUCTION}

From birth until 4.5 years of age he collected inked fingerprint impressions of a infant, compared them manually, and conjectured that it was possible to use fingerprints to identify children older than 2.5 years. Since Galton's study of fingerprinting young children, the digital capture and automated analysis of fingerprints have made considerable progress. The inkon-paper fingerprint acquisition process was largely superseded by live scanning methods that include a digital fingerprint image directly. Tedious manual comparison of fingerprints has been replaced by fast and reliable automatic methods of comparison. These technical developments, as well as emerging technologies involving children's identification, have reinvigorated the fingerprint research community's interest in investigating child fingerprinting, and recently led to a few feasibility studies on child fingerprint recognition. However, the consensus between fingerprint practitioners and the general public is that identifying young children 1 from their fingerprints is not feasible. On the other hand, biological evidence indicates that fingerprints are completely developed by the sixth month of fetal life, and are physiologically present at birth on human fingers. In addition, it is often assumed that fingerprints are I distinct, i.e. no two fingers, even of the same individual, have the same patterns, and (ii) permanent, i.e. they do not alter over an person's lifetime. Whereas the uniqueness and durability of fingerprints for adult fingerprints have been studied, there has been, to our knowledge, no systematic and comprehensive longitudinal research to answer the following fundamental questions concerning child fingerprints: 1) Do children's fingerprints have the distinctive characteristics required to identify a child uniquely? 2) What is the youngest age at which a child's fingerprints can be captured with sufficient fidelity to uniquely recognize the child? 3) Can a child's fingerprints be used to dependably recognize the child as he ages?

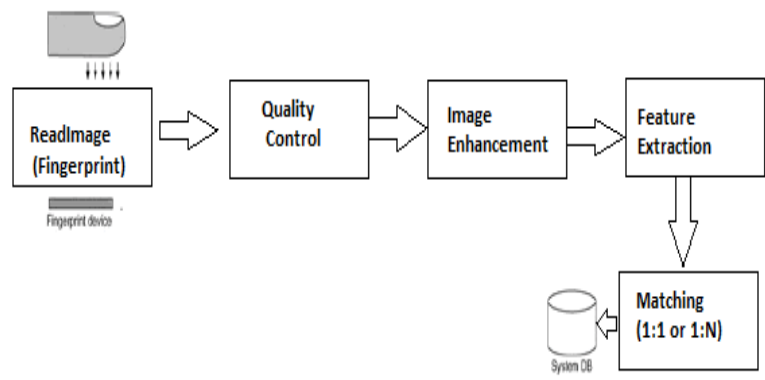

Figure 1: Proposed System 


\section{RELATED WORK}

Identification and recognition of children and infants have come to limelight in recent years. Some early work in identification of children less than 5 years, date to as early as 1939 when Louise et.al tried identification of infants using their palm prints[5]. In 2008, Weingaetar et al. compared performance of footprint against palm prints for 106 new-born kids. It was observed that palm prints were of better quality than food prints. Galton et al. in 1989 recorded inked fingerprint impression of children from 0-4.5 years and conjectured that minimum age for children to be identified through fingerprint is 2.5 years [10].

Recently, Jain et al. in 2015, collected fingerprint data of 206 children between $0-5$ years age over a span of an year in four different phases. Their work aims at investigating the persistence of fingerprint recognition. However, they only recorded thumb samples for every subject instead of all the fingers. Gottschlich tried to predict development in features of fingerprint with age. He studied fingerprint of 48 subjects when they were 615 years old and 17-34 years old respectively. His observations suggested that development of fingerprint depends on the gender specific growth pattern. So far, there has been no literary study to support the developmental changes in fingerprint for kids less than 5 years. Bharadwaj et. Al [11] assert that despite of challenges of capturing face in newborns such as variation in face expression, expressions and pose, etc. newborns can be distinctly identified through face biometric.

In [12] Bharadwaj et. al extracted facial features using auto-encoders and matched using distance metric learning for over 450 newborn subjects.

The most comprehensive study to date focused on the permanence of fingerprints was published in 2015 by researchers from Michigan State University [16]. While the works by Galton and Herschel focused on analyzing the variability (or invariability) of the physical ridge structure, MSU's study concentrates on the impact that changes in the digital representation of the ridge structure (i.e., fingerprint images) may have on the genuine matching scores of automatic recognition systems working with current live-scan imaging technology (i.e., ageing). This "digital" interpretation of fingerprints permanence is the same considered in the present article.

\section{PROPOSED SYSTEM}

An in-depth review of kid fingerprint recognition studies since Galton's initial investigation within the year 1899. Assortment of fingerprints of 309 kids (age range: $0-5$ years) in four totally different sessions over a 1 year amount. For our preliminary work, we have a tendency to had collected fingerprints of solely sixty six kids within the 0-6 months adulthood cluster in 2 totally different sessions 2-4 days apart. Systematic and rigorous performance analysis of kid fingerprint recognition accuracy over the one year amount. We have a tendency to show that progressive fingerprint recognition capture and recognition technology offers a viable answer for recognizing kids older than six months (98.9\% TAR at zero.1\% FAR). The popularity performance obtained is love that obtained on adult fingerprints in FVC 2004 DB1 A and DB2 A. Use of mixed-effects applied mathematics models to check the trend of real fingerprint similarity scores over the one year fundamental measure. We have a tendency to show that kid fingerprint recognition accuracy doesn't degrade over the one year time lapse in our information.

\section{METHODOLOGY}

1. To enhance image quality, we have a tendency to designed associate degree rule to reinforce baby fingerprint pictures made public as follows.

2. Foreground region containing the fingerprint is detected by thresholding the individual constituent values.

3. Fingerprint is split into overlapping patches of size $160 \times 160$ pixels. Every patch is input to a convolution neural network $(\mathrm{CNN})$ trained for estimating ridge flow within the fingerprint.

4. Given improved fingerprint images, we conduct both verification (1:1 comparison) and identification (1:N comparisons) experiments using a commercial fingerprint SDK.

5. The three fingerprints captured on the first day are implicit to be enrolled in the database (obtained during infant's first visit for vaccination) and each of the three prints captured on the day second are taken as the query prints (e.g., obtained, for example, during subsequent follow-up visits).

6. Similarity scores obtained by comparing a query print to the three enrolled templates for each of the two fingers (left and right thumbs) are combined (sum fusion rule) to compute the final similarity score.

7. To evaluate the verification performance, two different metrics are computed,

a. true accepts: number of subjects, which can be correctly verified to have been previously enrolled, and

b. false accepts: number of subjects, which are incorrectly verified as previously enrolled. false accept rate (FAR) and true accept rate (TAR) are then computed to measure how frequently true accepts and false accepts occur.

8. In the identification mode, the captured fingerprint is compared against a background database containing fingerprints of known subjects, and a candidate list of the top-K matches is retrieved from the database.

9. The percentage of fingerprint queries for which the mated fingerprint is retrieved amongst the top- $i$ candidates (rank-i) in the K-candidate list is used as the evaluation criteria.

\section{Image Enhancement Algorithm}

In computer graphics, the method of up the standard of a digitally hold on image by manipulating the image with code. It's quite straightforward, for instance, to form a picture lighter or darker, or to extend or decrease distinction. Programs specialized for image improvement area unit generally referred to as image 
editors. Following area unit the image improvement techniques:

1. Filtering with morphological operators.

2. Histogram equalization.

3. Noise removal using a Wiener filter.

4. Linear contrast adjustment.

5. Median filtering.

6. Un sharp mask filtering.

7. Contrast-limited adaptive histogram equalization (CLAHE)

8. Decor relation stretch.

\section{EXPERIMENTAL RESULTS}

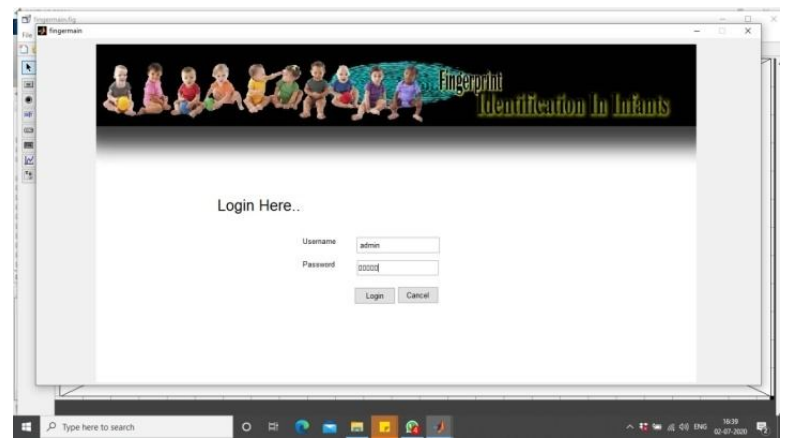

Figure 2: login page

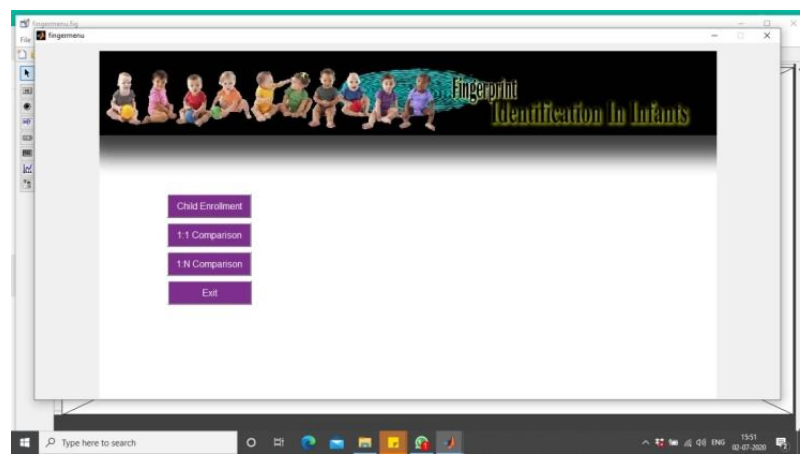

Figure 3: Home screen

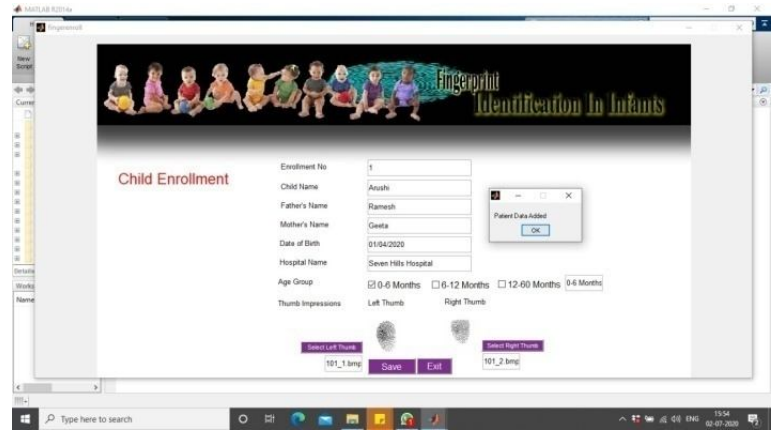

Figure 4: Child Enrollment

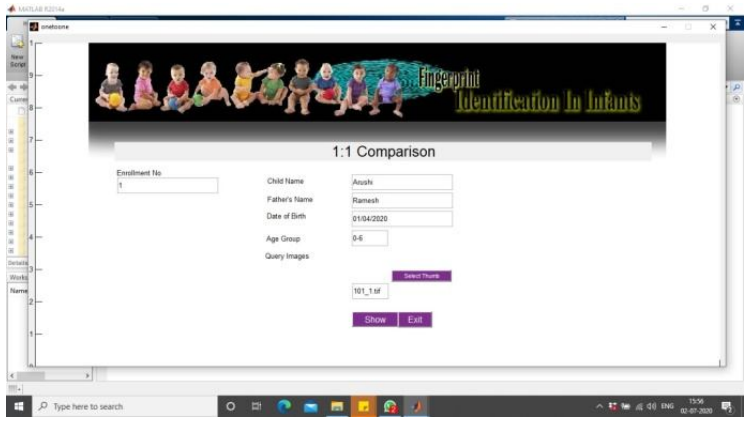

Figure 5: 1:1 Comparison

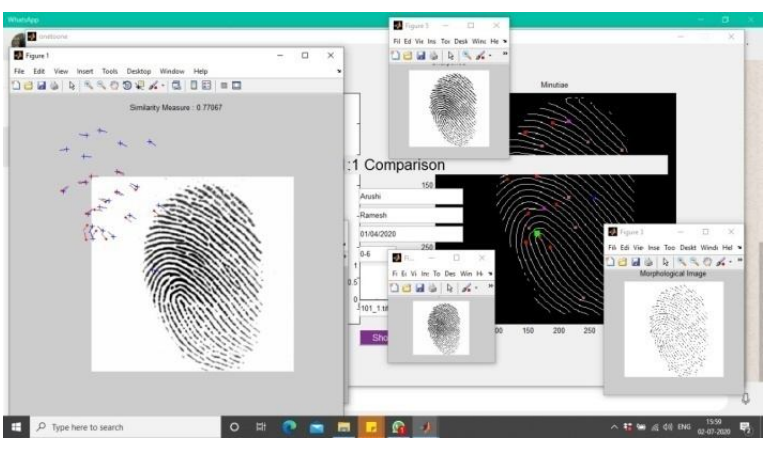

Figure 6: 1:1 Comparison analysis

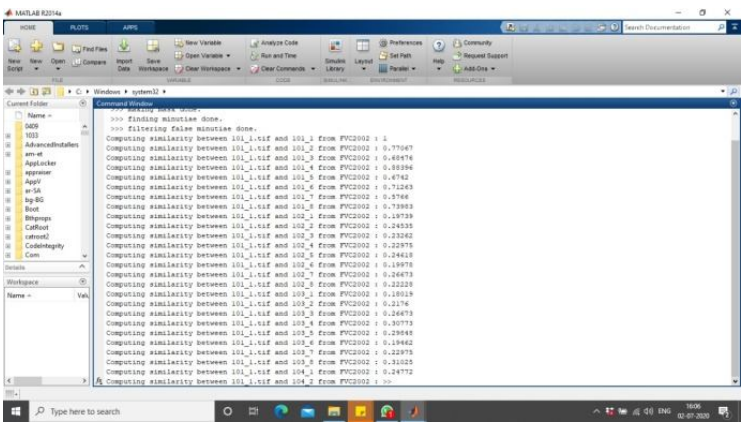

Figure 7: 1:M Comparison

\section{CONCLUSION}

We terminated that, essential step in automatic fingerprint matching is just too automatically and dependably extract minutiae from the input fingerprint images. Though, the performance of a minutiae extraction algorithmic program depends heavily on the standard of the input fingerprint images. so as to confirm that the performance of an automatic fingerprint identification/verification system would be robust with respect to the quality of the fingerprint images, it ought to be essential to include a fingerprint enhancement algorithmic program within the minutiae extraction module. In our case, the quality of the image is actually good, and that we will not have to be compelled to enhance our image. 


\section{REFERENCES}

[1] A. K. Jain, S. S. Arora, L. Best-Rowden, K. Cao, P. S. Sudhish, A. Bhatnagar, and Y. Koda, "Giving infants an identity: Fingerprint sensing and recognition," in Proceedings of the Eighth International Conference on Information and Communication Technologies and Development. ACM, 2016, pp. 29-32.

[2] F. Galton, Finger prints. Macmillan and Company, 1892.

[3] Y. Koda, T. Higuchi, and A. K. Jain, "Advances in capturing child fingerprints: A high resolution CMOS image sensor with SLDR method," in International Conference of the Biometrics Special Interest Group (BIOSIG), 2016.

[4] F. Galton, "Finger Prints of Young Children," British Association for the Advancement of Science, vol. 69, pp. 868-869, 1899.

[5] MORGAN, L. E., AND PAULS, F. Palm prints for infant identification. AJN The American Journal of Nursing 39, 8 (1939), 866-868.

[6] F. Rahmun and O. Bausinger, "Best Practice Fingerprint Enrolment Standards - European Visa Information System," in NIST International Biometric Performance Testing Conference, 2010, http://biometrics.nist.gov/cs

links/ibpc2010/pdfs/Rahmun Fares BausingerOliver 20100303 BestPracticeFingerprintEnrolmentVIS.pdf.

[7] J. Schneider, "Quantifying the dermatoglyphic growth patterns in children through adolescence," Ultra-Scan Corporation, Tech. Rep. FR00A178000-1, 2010.

[8] "Fingerprint Recognition for Children," Joint Research Center of the European Commission, Tech. Rep., 2013, https://ec.europa.eu/jrc/en/publication/eurscientific-and-technicalresearch-reports/fingerprintrecognition-children.

[9] H. Cummins and C. Midlo, Finger prints, Palms and Soles: An Introduction to Dermatoglyphics. Dover Publications New York, 1961, vol. 319.
[10] JAIN, A. K., ARORA, S. S., BEST-ROWDEN, L., CAO, K., SUDHISH, P. S., BHATNAGAR, A., AND KODA, Y. Giving infants an identity: Fingerprint sensing and recognition. In proceedings of the eighth international conference on information and communication technologies and development (2016), ACM, p.29.

[11] BHARADWAJ, S., BHATT, H. S., SINGH, R., VATSA, M., AND SINGH, S. K. Face recognition for newborns: A preliminary study. In Biometrics: Theory Applications and Systems (BTAS), 2010 Fourth IEEE International Conference on (2010), IEEE, pp. 1-6.

[12] BHARADWAJ, S., BHATT, H. S., VATSA, M., AND SINGH, R. Domain specific learning for newborn face recognition. IEEE Transactions on Information Forensics and Security 11, 7 (2016), 1630-1641.

[13] W. J. Herschel, The Origins of Fingerprinting. Oxford University Press, 1916.

[14] S. Pankanti, S. Prabhakar, and A. K. Jain, "On the individuality of fingerprints," IEEE Transactions on Pattern Analysis and Machine Intelligence, vol. 24, no. 8, pp. 1010-1025, 2002.

[15] Y. Zhu, S. C. Dass, and A. K. Jain, "Statistical models for assessing the individuality of fingerprints," IEEE Transactions on Information Forensics and Security, vol. 2, no. 3, pp. 391-401, 2007.

[16] S. Yoon and A. K. Jain, "Longitudinal study of fingerprint recognition," Proc. Nat. Acad. Sci. USA, vol. 112, no. 28, pp. 8555-8560, 2015. 